\title{
Search for Standard Model Higgs boson in di-tau final state at the Tevatron
}

\author{
Pierluigi Totaro*† \\ INFN-Sezione di Trieste \\ E-mail: totarodfnal.gov
}

\begin{abstract}
This paper reports the searches for the Standard Model (SM) Higgs boson in the di-tau final state, performed by CDF and $\mathrm{D} \emptyset$ collaborations at the Tevatron $p \bar{p}$ collider. The presented results refer respectively to $2.3 \mathrm{fb}^{-1}$ and $4.9^{-1}$ of data collected at $\sqrt{s}=1.96 \mathrm{TeV}$, and involve several signal processes, which are examined simultaneously: $\mathrm{gg} \rightarrow \mathrm{H} \rightarrow \tau \tau, \mathrm{WH}(\rightarrow \tau \tau), \mathrm{ZH}(\rightarrow \tau \tau), \mathrm{HZ}(\rightarrow \tau \tau)$ and $\mathrm{qHq} \rightarrow \mathrm{q} \tau \tau \mathrm{q}^{\prime}$. Both the analyses are performed by looking for Higgs candidates events where one $\tau$ decays leptonically and the other one is reconstructed by its hadronic decay products. In addition, one or more energetic calorimeter jets are required in the final state.

The sensitivity of the searches is enhanced by employing multivariate techniques, based in both $\mathrm{CDF}$ and $\mathrm{D} \emptyset$ on sets of Boosted Decision Trees trained to provide the best separation between Higgs signal and the dominant sources of background. No significant excess of events above the SM background is observed, so the measurements are used to set $95 \%$ confidence level (C.L.) exclusion limits on the Higgs production cross section.
\end{abstract}

35th International Conference of High Energy Physics

July 22-28, 2010

Paris, France

\footnotetext{
* Speaker.

$\dagger$ On behalf of the CDF and DØ collaborations.
} 


\section{Introduction}

In the Standard Model of Particle Physics the Higgs mechanism explains how fundamental particles may acquire mass, through the spontaneous breaking of the electroweak symmetry. This mechanism implies the prediction of a heavy neutral particle, the Higgs boson, whose existence has not yet been experimentally confirmed.

The searches carried out by CDF and DØ collaborations explore the entire Higgs boson mass range allowed by the theoretical and experimental constraints, $100-200 \mathrm{GeV} / \mathrm{c}^{2}$. The dominant production mode at the Tevatron $p \bar{p}$ collider is gluon-gluon fusion, while the main decay process for masses below $135 \mathrm{GeV} / \mathrm{c}^{2}$ is into a $b \bar{b}$ pair. However, the process $\mathrm{gg} \rightarrow \mathrm{H} \rightarrow b \bar{b}$ is experimentally prohibitive due to the overwhelming QCD dijet background, and thus searches have predominantly focused on the associative production with a $\mathrm{W}$ or a $\mathrm{Z}$ boson, with the Higgs subsequently decaying into $\mathrm{b}$ quarks and the vector bosons mainly identified through their leptonic decay products.

The search sensitivity in the low mass region can be increased by looking at the complementary Higgs channels characterized by tau pairs in the final state. Although the branching ratio (B.R.) is one order of magnitude smaller thant the decay into $b$ quarks, several production processes can be studied simultaneously, including direct production and vector boson fusion (VBF). In addition, a lot of signal acceptance in the associated production can ben recovered by extending the search to the hadronic decay of $\mathrm{W}$ and $\mathrm{Z}$ bosons.

The analyses presented in this paper have been performed using $2.3 \mathrm{fb}^{-1}$ and $4.9 \mathrm{fb}^{-1}$ of data collected by the CDF[ [3] and DØ[円] experiments, respectively. A detailed description of the detectors can be found elsewhere[1]纤.

\section{Event selection and search strategies}

Tau leptons are short lived particles which can be detected only through their visible decay products. Hadronic decays, denoted as $\tau_{\mathrm{h}}$, are of the form $\tau \rightarrow X_{\mathrm{h}} v_{\tau}$, where $X_{\mathrm{h}}$ is a system of hadrons, mainly composed of charged and neutral pions. The corresponding B.R. is about $65 \%$ and the typical signature in the detector is made up of narrow calorimeter clusters matched to low multiplicity reconstructed tracks. The remaining channels are represented by the leptonic decays into muons $\left(\tau_{\mu}\right)$ or electrons $\left(\tau_{e}\right)$, through the process $\tau \rightarrow l v_{l} v_{\tau}$.

$\mathrm{D} \emptyset$ experiment limits the search to the $\tau_{\mathrm{h}} \tau_{\mu}$ combination, while CDF also covers the $\tau_{\mathrm{h}} \tau_{e}$ channel: the choice of the "leptonic+hadronic decay" final state represents the best compromise between the high B.R. of the hadronic tau decay and the large background suppression provided by the request of an electron or a muon.

Both CDF and D $\varnothing$ select events collected by dedicated triggers and apply sets of quality offline requirements to reconstruct high transverse momentum $\left(p_{\mathrm{T}}\right)$ leptons. In particular muons are identified by hits in the outer muon detectors, geometrically matched to tracks reconstructed in the inner tracking system; electron candidates consist of tracks associated to electromagnetic calorimeter clusters. Both experiments identify hadronically decaying taus by employing sophisticated multivariate algorithms, optimized to separate real taus from quark/gluon jets: DØ has developed an algorithm based on neural networks, while CDF has implemented a set of Boosted Decision Trees (BDTs). 


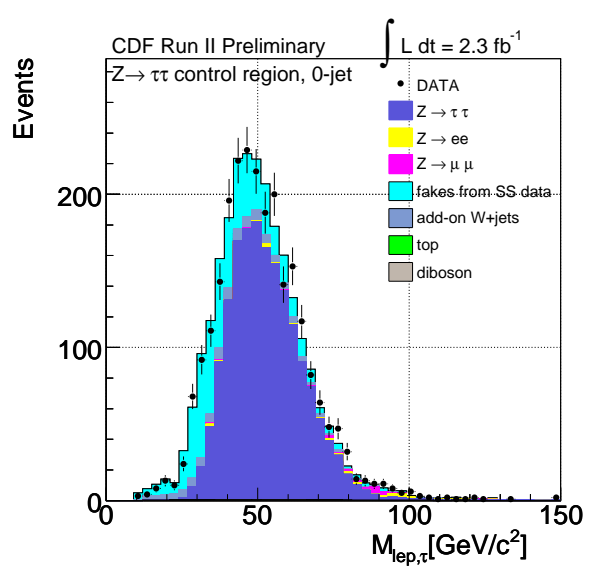

(a)

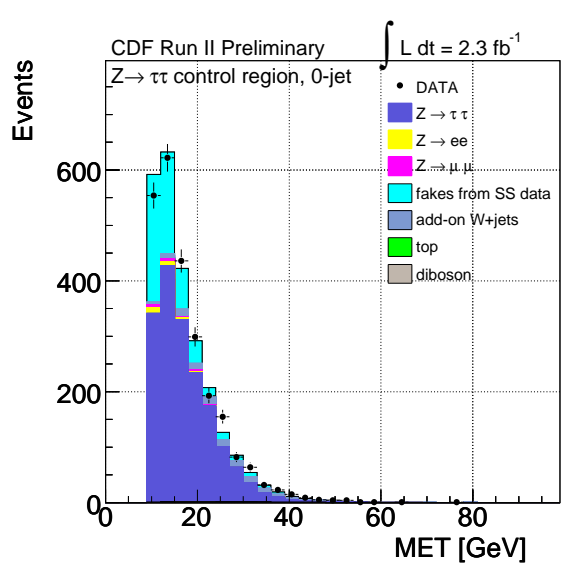

(b)

Figure 1: The CDF analysis showing the expected and observed event yields in the $\mathrm{Z} \rightarrow \tau \tau$ control sample $\left(\mathbb{E}_{T}>10 \mathrm{GeV}\right.$ and $\mathrm{M}_{\mathrm{T}}\left(\mathrm{lep}, \mathbb{G}_{T}\right)<60 \mathrm{GeV} / \mathrm{c}^{2}$, no jets): (a)lepton+tau invariant mass; (b)missing transverse energy.

The two reconstructed leptons need to be spatially well separated, come from the same interaction point and have opposite charges. In addition, one (CDF) or more (CDF and DØ) calorimeter jets must be present in the final state, thus maximizing the sensitivity to VBF and associated production where gauge bosons are allowed to decay hadronically. Jets are reconstructed using fixed cone algorithms and the energy is opportunely corrected for instrumental effects up to the particle level. The transverse component of the sum of the neutrinos' momenta is estimated by calculating the calorimeter energy imbalance in the transverse plane, $\mathbb{E}_{T}=\left|\mathbf{E}_{T}\right|$.

The main sources of physics processes which enter in the event selection include $Z / \gamma^{*} \rightarrow \tau \tau$, $t \bar{t}$ and diboson events, which are fully estimated with Monte Carlo (MC) simulations. A data based procedure is followed both by $\mathrm{CDF}$ and $\mathrm{D} \emptyset$ for the estimation of the background processes which come from misidentified leptons, mainly arising from jet reconstructed as hadronic taus. Background modeling is tested in several control samples, where a good agreement is found between the estimated and the observed rates and kinematic distributions, as reported in figure 1 for the CDF analysis.

Due to the fact that the event selection is sensitive to the number of reconstructed jets in the final state, the main sources of systematic uncertainties which affect the measurement are those related to jet reconstruction and energy scale definition.

After applying the selection, no significant excess above the SM is noticed in any kinematic distribution of the signal regions, and exclusion limits on the Higgs production cross section are then placed. The search sensitivity is increased in CDF and DØ by employing BDTs to collect the discriminating power of different variables into one single distribution, which is then used in the limit calculation. Results are shown in figure 2, for the Higgs mass range between 100 and 150 $\mathrm{GeV} / \mathrm{c}^{2}$ : at the mass hypothesis of $115 \mathrm{GeV} / \mathrm{c}^{2}$ the observed(expected) limit calculated by CDF and $\mathrm{D} \emptyset$ are 27.9(24.5) and 27.0(15.9) times larger then the SM prediction, with $2.3 \mathrm{fb}^{-1}$ and 4.9 $\mathrm{fb}^{-1}$ of data, respectively. 


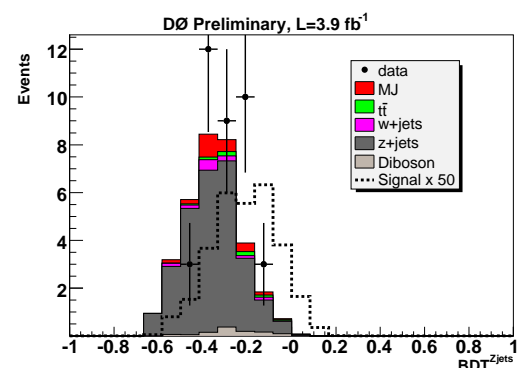

(a)

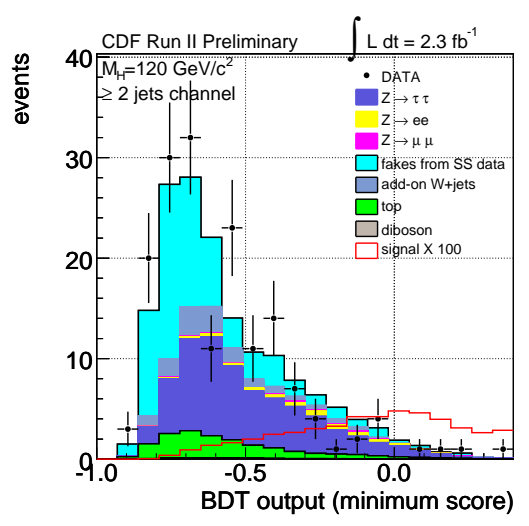

(c)

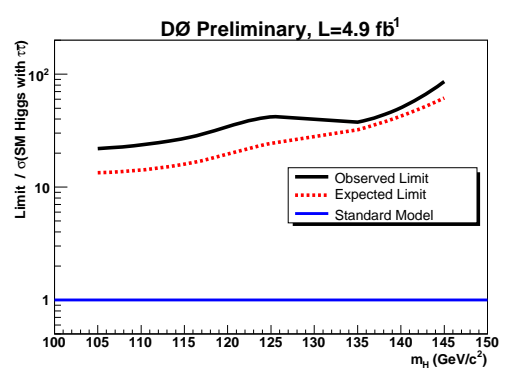

(b)

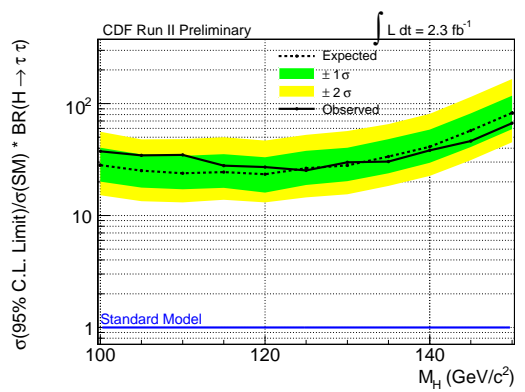

(d)

Figure 2: (a)D $\varnothing$ BDT final discriminant distribution for $m_{\mathrm{H}}=115 \mathrm{GeV} / \mathrm{c}^{2}$ in the $\tau \tau+2$ jets final state; (b) Ratio of the expected and observed 95\% C.L. upper limit to the SM Higgs boson prediction, as a function of the Higgs boson mass, for the DØ experiment; (c) CDF BDT final discriminant distribution for $m_{\mathrm{H}}=115$ $\mathrm{GeV} / \mathrm{c}^{2}$ in the $\tau \tau+2$ jets final state; (d) Ratio of the expected and observed 95\% C.L. upper limit to the SM Higgs boson prediction, for the CDF experiment.

\section{Conclusions}

We have presented the search for the SM Higgs in the di-tau final state performed at the Tevatron experiments. No evidence for a signal has been reported and 95\% C.L. exclusion limits have been set. The results, which contribute to increase the overall Tevatron sensitivity in the low mass region searches [], are expected to further improve as well as new data are included and additional advanced analysis techniques are implemented.

\section{References}

[1] CDF Collaboration, D.Acosta et al., Phys. Rev. D71, 032001(2005)

[2] DØ Collaboration, V.M.Abazov et al., Nucl. Instrum. Methods Phys. Res. A 565, 463(2006)

[3] CDF Collaboration, T.Aaltonen et al., CDF Conference Note 10133

[4] DØ Collaboration, V.M.Abazov et al., DØ Conference Note 5845

[5] CDF and DØ Collaborations, Combined CDF and D0 Upper Limits on Standard Model Higgs-Boson Production with up to $6.7 \mathrm{fb}-1$ of Data, arXiv:1007.4587v1 